\title{
QUANTITATIVE VERSION OF THE BISHOP-PHELPS-BOLLOBÁS THEOREM FOR OPERATORS WITH VALUES IN A SPACE WITH THE PROPERTY $\beta$
}

\begin{abstract}
V. Kadets, M. Soloviova. Quantitative version of the Bishop-Phelps-Bollobás theorem for operators with values in a space with the property $\beta$, Mat. Stud. 47 (2017), 71-90.

The Bishop-Phelps-Bollobás property for operators deals with simultaneous approximation of an operator $T$ and a vector $x$ at which $T: X \rightarrow Y$ nearly attains its norm by an operator $F$ and a vector $z$, respectively, such that $F$ attains its norm at $z$. We study the possible estimates from above and from below for parameters that measure the rate of approximation in the Bishop-Phelps-Bollobás property for operators for the case of $Y$ having the property $\beta$ of Lindenstrauss.
\end{abstract}

1. Introduction. In this paper $X, Y$ are real Banach spaces, $L(X, Y)$ is the space of all bounded linear operators $T: X \rightarrow Y, L(X)=L(X, X), X^{*}=L(X, \mathbb{R}), B_{X}$ and $S_{X}$ denote the closed unit ball and the unit sphere of $X$, respectively. A functional $x^{*} \in X^{*}$ attains its norm, if there is $x \in S_{X}$ with $x^{*}(x)=\left\|x^{*}\right\|$. The Bishop-Phelps theorem [3] (see also [8, Chapter 1, p. 3]) says that the set of norm-attaining functionals is always dense in $X^{*}$. In [4] B. Bollobás remarked that in fact the Bishop-Phelps construction allows to approximate at the same time a functional and a vector at which it almost attains the norm. Nowadays this very useful fact is called the Bishop-Phelps-Bollobás theorem. Recently, two moduli have been introduced [5] which measure, for a given Banach space, what is the best possible Bishop-Phelps-Bollobás theorem in that space. We will use the following notation:

$$
\Pi(X):=\left\{\left(x, x^{*}\right) \in X \times X^{*}:\|x\|=\left\|x^{*}\right\|=x^{*}(x)=1\right\} .
$$

Definition 1 (Bishop-Phelps-Bollobás moduli, [5]). Let $X$ be a Banach space. The Bishop-Phelps-Bollobás modulus of $X$ is the function $\Phi_{X}:(0,2) \longrightarrow \mathbb{R}^{+}$such that given $\varepsilon \in(0,2), \Phi_{X}(\varepsilon)$ is the infimum of those $\delta>0$ satisfying that for every $\left(x, x^{*}\right) \in B_{X} \times B_{X^{*}}$ with $x^{*}(x)>1-\varepsilon$, there is $\left(y, y^{*}\right) \in \Pi(X)$ with $\|x-y\|<\delta$ and $\left\|x^{*}-y^{*}\right\|<\delta$. Substituting $\left(x, x^{*}\right) \in S_{X} \times S_{X^{*}}$ instead of $\left(x, x^{*}\right) \in B_{X} \times B_{X^{*}}$ in the above sentence, we obtain the definition of the spherical Bishop-Phelps-Bollobás modulus $\Phi_{X}^{S}(\varepsilon)$.

Evidently, $\Phi_{X}^{S}(\varepsilon) \leqslant \Phi_{X}(\varepsilon)$. There is a common upper bound for $\Phi_{X}(\cdot)$ (and so for $\Phi_{X}^{S}(\cdot)$ ) for all Banach spaces which is actually sharp. Namely [5], for every Banach space $X$ and every $\varepsilon \in(0,2)$ one has $\Phi_{X}(\varepsilon) \leqslant \sqrt{2 \varepsilon}$. In other words, this leads to the following improved version of the Bishop-Phelps-Bollobás theorem.

2010 Mathematics Subject Classification: 46B04, 46B20, 46B22, 47A30.

Keywords: Bishop-Phelps-Bollobás theorem; norm-attaining operators; property $\beta$ of Lindenstrauss.

doi:10.15330/ms.47.1.71-90

(C) V. Kadets, M. Soloviova, 2017 
Proposition 1 ([5, Corollary 2.4]). Let $X$ be a Banach space and $0<\varepsilon<2$. Suppose that $x \in B_{X}$ and $x^{*} \in B_{X^{*}}$ satisfy $x^{*}(x)>1-\varepsilon$. Then, there exists $\left(y, y^{*}\right) \in \Pi(X)$ such that $\|x-y\|<\sqrt{2 \varepsilon}$ and $\left\|x^{*}-y^{*}\right\|<\sqrt{2 \varepsilon}$.

The sharpness of this version is demonstrated in [5, Example 2.5] by just considering $X=\ell_{1}^{(2)}$, the two-dimensional real $\ell_{1}$ space. For a uniformly non-square Banach space $X$ one has $\Phi_{X}(\varepsilon)<\sqrt{2 \varepsilon}$ for all $\varepsilon \in(0,2)$ ([5, Theorem 5.9], [7, Theorem 2.3]). A quantifcation of this inequality in terms of a parameter that measures the uniform non-squareness of $X$ was given in [6, Theorem 3.3].

Lindenstrauss in [12] examined the extension of the Bishop-Phelps theorem on denseness of the family of norm-attaining scalar-valued functionals on a Banach space, to vector-valued linear operators. He introduced the property $\beta$, which is possessed by polyhedral finitedimensional spaces, and by any subspace of $\ell_{\infty}$ that contains $c_{0}$.

Definition 2. A Banach space $Y$ is said to have the property $\beta$ if there are two sets $\left\{y_{\alpha}: \alpha \in\right.$ $\Lambda\} \subset S_{Y},\left\{y_{\alpha}^{*}: \alpha \in \Lambda\right\} \subset S_{Y}^{*}$ and $0 \leqslant \rho<1$ such that the following conditions hold

(i) $y_{\alpha}^{*}\left(y_{\alpha}\right)=1$,

(ii) $\left|y_{\alpha}^{*}\left(y_{\gamma}\right)\right| \leqslant \rho$ if $\alpha \neq \gamma$,

(iii) $\|y\|=\sup \left\{\left|y_{\alpha}^{*}(y)\right|: \alpha \in \Lambda\right\}$, for all $y \in Y$.

Denote for short by $\beta(Y) \leqslant \rho$ that a Banach space $Y$ has the property $\beta$ with parameter $\rho \in(0,1)$. Obviously, if $\rho_{1} \leqslant \rho_{2}<1$ and $\beta(Y) \leqslant \rho_{1}$, then $\beta(Y) \leqslant \rho_{2}$. If $Y$ has the property $\beta$ with parameter $\rho=0$, we will write $\beta(Y)=0$.

Lindenstrauss proved that if a Banach space $Y$ has the property $\beta$, then for any Banach space $X$ the set of norm attaining operators is dense in $L(X, Y)$. It was proved later by J. Partington ([10]) that every Banach space can be equivalently renormed to have the property $\beta$.

In 2008, Acosta, Aron, García and Maestre in [1] introduced the following Bishop-PhelpsBollobás property as an extension of the Bishop-Phelps-Bollobás theorem to the vectorvalued case.

Definition 3. A couple of Banach spaces $(X, Y)$ is said to have the Bishop-Phelps-Bollobás property for operators if for any $\delta>0$ there exists a $\varepsilon(\delta)>0$, such that for every operator $T \in S_{L(X, Y)}$, if $x \in S_{X}$ and $\|T(x)\|>1-\varepsilon(\delta)$, then there exist $z \in S_{X}$ and $F \in S_{L(X, Y)}$ satisfying $\|F(z)\|=1,\|x-z\|<\delta$ and $\|T-F\|<\delta$.

In [1, Theorem 2.2] it was proved that if $Y$ has the property $\beta$, then for any Banach space $X$ the pair $(X, Y)$ has the Bishop-Phelps-Bollobás property for operators. In this article we introduce an analogue of the Bishop-Phelps-Bollobás moduli for the vector-valued case.

Definition 4. Let $X, Y$ be Banach spaces. The Bishop-Phelps-Bollobás modulus (spherical Bishop-Phelps-Bollobás modulus) of a pair $(X, Y)$ is the function $\Phi(X, Y, \cdot):(0,1) \longrightarrow \mathbb{R}^{+}$ $\left(\Phi^{S}(X, Y, \cdot):(0,1) \longrightarrow \mathbb{R}^{+}\right)$whose value in point $\varepsilon \in(0,1)$ is defined as the infimum of those $\delta>0$ such that for every $(x, T) \in B_{X} \times B_{L(X, Y)}\left((x, T) \in S_{X} \times S_{L(X, Y)}\right.$ respectively) with $\|T(x)\|>1-\varepsilon$, there is $(z, F) \in S_{X} \times S_{L(X, Y)}$ with $\|F(z)\|=1,\|x-z\|<\delta$ and $\|T-F\|<\delta$. 
Under the notation

$$
\begin{gathered}
\Pi_{\varepsilon}(X, Y)=\{(x, T) \in X \times L(X, Y):\|x\| \leqslant 1,\|T\| \leqslant 1,\|T(x)\|>1-\varepsilon\}, \\
\Pi_{\varepsilon}^{S}(X, Y)=\{(x, T) \in X \times L(X, Y):\|x\|=\|T\|=1,\|T(x)\|>1-\varepsilon\}, \\
\Pi(X, Y)=\{(x, T) \in X \times L(X, Y):\|x\|=1,\|T\|=1,\|T(x)\|=1\},
\end{gathered}
$$

the definition can be rewritten as follows

$$
\begin{aligned}
\Phi(X, Y, \varepsilon) & =\sup _{(x, T) \in \Pi_{\varepsilon}(X, Y)} \inf _{(z, F) \in \Pi(X, Y)} \max \{\|x-z\|,\|T-F\|\}, \\
\Phi^{S}(X, Y, \varepsilon) & =\sup _{(x, T) \in \Pi_{\varepsilon}^{S}(X, Y)} \inf _{(z, F) \in \Pi(X, Y)} \max \{\|x-z\|,\|T-F\|\} .
\end{aligned}
$$

Evidently, $\Phi^{S}(X, Y, \varepsilon) \leqslant \Phi(X, Y, \varepsilon)$, so any estimation from above for $\Phi(X, Y, \cdot)$ is also valid for $\Phi^{S}(X, Y, \cdot)$ and any estimation from below for $\Phi^{S}(X, Y, \cdot)$ is applicable to $\Phi(X, Y, \cdot)$. Also the following result is immediate.

Remark 1. Let $X, Y$ be Banach spaces, $\varepsilon_{1}, \varepsilon_{2}>0$ with $\varepsilon_{1}<\varepsilon_{2}$. Then $\Pi_{\varepsilon_{1}}(X, Y) \subset$ $\Pi_{\varepsilon_{2}}(X, Y)$ and $\Pi_{\varepsilon_{1}}^{S}(X, Y) \subset \Pi_{\varepsilon_{2}}^{S}(X, Y)$. Therefore, $\Phi(X, Y, \varepsilon)$ and $\Phi^{S}(X, Y, \varepsilon)$ do not decrease as $\varepsilon$ increases.

Notice that a couple $(X, Y)$ has the Bishop-Phelps-Bollobás property for operators if and only if $\Phi(X, Y, \varepsilon) \underset{\varepsilon \rightarrow 0}{\longrightarrow} 0$.

The aim of the paper is to estimate the Bishop-Phelps-Bollobás modulus for operators which act to a Banach space with the property $\beta$. This paper is organized as follows. After the Introduction, in Section 2 we will provide an estimation from above for $\Phi(X, Y, \varepsilon)$ for $Y$ possessing the property $\beta$ of Lindenstrauss (Theorem 1) and an improvement for the case of $X$ being uniformly non-square (Theorem 2). Section 3 is devoted to estimations of $\Phi(X, Y, \varepsilon)$ from below and related problems. As a bi-product of these estimations we obtain an interesting effect (Theorem 6) that $\Phi(X, Y, \varepsilon)$ is not continuous with respect to the variable $Y$. In Section 4 we consider a modification of the above moduli which appear if one approximates by pairs $(y, F)$ with $\|F\|=\|F y\|$ without requiring $\|F\|=1$. Finally, in a very short Section 5 we speak about a natural question which we did not succeed to solve.

2. Estimation from above. Our first result is the upper bound of the Bishop-PhelpsBollobás moduli for the case when the range space has the property $\beta$ of Lindenstrauss.

Theorem 1. Let $X$ and $Y$ be Banach spaces such that $\beta(Y) \leqslant \rho$. Then for every $\varepsilon \in(0,1)$

$$
\Phi^{S}(X, Y, \varepsilon) \leqslant \Phi(X, Y, \varepsilon) \leqslant \min \left\{\sqrt{2 \varepsilon} \sqrt{\frac{1+\rho}{1-\rho}}, 2\right\} .
$$

The above result is a quantification of [1, Theorem 2.2] which states that if $Y$ has the property $\beta$, then for any Banach space $X$ the pair $(X, Y)$ has the Bishop-Phelps-Bollobás property for operators. The construction is borrowed from the demonstration of $[1$, Theorem 2.2], but in order to obtain (1) we have to take care about details and need some additional work. At first, we have to modify a little bit the original results of Phelps about approximation of a functional $x^{*}$ and a vector $x$.

Proposition 2 ([13], Corollary 2.2). Let $X$ be a real Banach space, $x \in B_{X}, x^{*} \in S_{X^{*}}$, $\eta>0$ and $x^{*}(x)>1-\eta$. Then for any $k \in(0,1)$ there exist $\zeta^{*} \in X^{*}$ and $y \in S_{X}$ such that $\zeta^{*}(y)=\left\|\zeta^{*}\right\|,\|x-y\|<\frac{\eta}{k},\left\|x^{*}-\zeta^{*}\right\|<k$. 
For our purposes we need an improvement which allows to take any $x^{*} \in B_{X^{*}}$.

Lemma 1. Let $X$ be a real Banach space, $x \in B_{X}, x^{*} \in B_{X^{*}}, \varepsilon \in(0,1)$ and $x^{*}(x)>1-\varepsilon$. Then for any $k \in(0,1)$ there exist $y^{*} \in X^{*}$ and $z \in S_{X}$ such that

$$
y^{*}(z)=\left\|y^{*}\right\|, \quad\|x-z\|<\frac{1-\frac{1-\varepsilon}{\left\|x^{*}\right\|}}{k}, \quad\left\|x^{*}-y^{*}\right\|<k\left\|x^{*}\right\| .
$$

Moreover, for any $\tilde{k} \in[\varepsilon / 2,1)$ there exist $z^{*} \in S_{X^{*}}$ and $z \in S_{X}$ such that

$$
z^{*}(z)=1, \quad\|x-z\|<\frac{\varepsilon}{\tilde{k}}, \quad\left\|x^{*}-z^{*}\right\|<2 \tilde{k} .
$$

Proof. We have that $\frac{x^{*}}{\left\|x^{*}\right\|}(x)>1-\eta$ for $\eta=1-\frac{1-\varepsilon}{\left\|x^{*}\right\|}$ and we can apply Proposition 2. So, for any $k \in(0,1)$ there exist $\zeta^{*} \in X^{*}$ and $z \in S_{X}$ such that

$$
\zeta^{*}(z)=\left\|\zeta^{*}\right\|, \quad\|x-z\|<\frac{\eta}{k}, \quad\left\|\frac{x^{*}}{\left\|x^{*}\right\|}-\zeta^{*}\right\|<k .
$$

In order to get (2) it remains to introduce $y^{*}=\left\|x^{*}\right\| \cdot \zeta^{*}$. This functional also attains its norm at $z$ and $\left\|x^{*}-y^{*}\right\|=\left\|x^{*}\right\| \cdot\left\|\frac{x^{*}}{\left\|x^{*}\right\|}-\zeta^{*}\right\|<k\left\|x^{*}\right\|$. In order to demonstrate the "moreover" part, take $k=\frac{\tilde{k}\left(\left\|x^{*}\right\|-(1-\varepsilon)\right)}{\varepsilon\left\|x^{*}\right\|}$.

The inequality $\left\|x^{*}\right\| \geqslant x^{*}(x)>1-\varepsilon$ implies that $k>0$. On the other hand, $k=$ $\tilde{k}\left(\frac{1}{\varepsilon}-\frac{(1-\varepsilon)}{\varepsilon\left\|x^{*}\right\|}\right) \leqslant \tilde{k}\left(\frac{1}{\varepsilon}-\frac{(1-\varepsilon)}{\varepsilon}\right)=\tilde{k}<1$, so for this $k$ we can find $y^{*} \in X^{*}$ and $z \in S_{X}$ such that (2) holds true. Denote $z^{*}=\frac{y^{*}}{\left\|y^{*}\right\|}$. Then $\|x-z\|<\varepsilon / \tilde{k}$ and

$$
\begin{gathered}
\left\|x^{*}-z^{*}\right\| \leqslant\left\|x^{*}-y^{*}\right\|+\left\|y^{*}-z^{*}\right\| \leqslant\left\|x^{*}-y^{*}\right\|+\left|1-\left\|y^{*}\right\|\right| \leqslant \\
\leqslant\left\|x^{*}-y^{*}\right\|+\left|1-\left\|x^{*}\right\|+\left\|x^{*}\right\|-\left\|y^{*}\right\|\right| \leqslant 2\left\|x^{*}-y^{*}\right\|+1-\left\|x^{*}\right\| .
\end{gathered}
$$

So, we have

$$
\left\|x^{*}-z^{*}\right\|<2 k\left\|x^{*}\right\|+1-\left\|x^{*}\right\|=\frac{2 \tilde{k} \cdot\left(\left\|x^{*}\right\|-(1-\varepsilon)\right)}{\varepsilon}+1-\left\|x^{*}\right\| \leqslant 2 \tilde{k} .
$$

The latter inequality holds, since the function $f(t)=\frac{2 \tilde{k} \cdot(t-(1-\varepsilon))}{\varepsilon}+1-t$ with $t \in(1-\varepsilon, 1)$, is increasing when $\tilde{k} \geqslant \varepsilon / 2$, so $\max f=f(1)=2 \tilde{k}$.

Remark 2. One can easily see that for $\tilde{k}<\frac{\varepsilon}{2}$ the "moreover" part with (1) is trivially true (and is not sharp) because in this case the inequality $\|x-z\| \leqslant \frac{\varepsilon}{\tilde{k}}$ is weaker than the triangle inequality $\|x-z\| \leqslant 2$, so one can just use the density of the set of norm-attaining functionals in order to get the desired $\left(z, z^{*}\right) \in \Pi(X)$ with $\left\|x^{*}-z^{*}\right\|<2 \tilde{k}$.

Proof of Theorem 1. We will use the notations $\left\{y_{\alpha}: \alpha \in \Lambda\right\} \subset S_{Y}$ and $\left\{y_{\alpha}^{*}: \alpha \in \Lambda\right\} \subset S_{Y}^{*}$ from Definition 2 of the property $\beta$.

Consider $T \in B_{L(X, Y)}$ and $x \in B_{X}$ such that $\|T x\|>1-\varepsilon$. According to (iii) of Definition 2, there is $\alpha_{0} \in \Lambda$ such that $\left|y_{\alpha_{0}}^{*}(T x)\right|>1-\varepsilon$. By Lemma 1 , for any $k \in\left[\frac{\varepsilon}{2}, 1\right)$ and for any $\delta>0$ there exist $z^{*} \in S_{X^{*}}$ and $z \in S_{X}$ such that $\left|z^{*}(z)\right|=1,\|z-x\|<\varepsilon / k$ and $\left\|z^{*}-T^{*}\left(y_{\alpha_{0}}^{*}\right)\right\|<2 k$. 
For $\eta=2 k \frac{\rho}{1-\rho}$ let us introduce the following operator $S \in L(X, Y)$

$$
S(v)=T(v)+\left[(1+\eta) z^{*}(v)-\left(T^{*} y_{\alpha_{0}}^{*}\right)(v)\right] y_{\alpha_{0}} .
$$

Remark, that for all $y^{*} \in Y^{*}$

$$
S^{*}\left(y^{*}\right)=T^{*}\left(y^{*}\right)+\left[(1+\eta) z^{*}-T^{*} y_{\alpha_{0}}^{*}\right] y^{*}\left(y_{\alpha_{0}}\right) .
$$

According to (iii) of Definition 2 the set $\left\{y_{\alpha}^{*}: \alpha \in \Lambda\right\}$ is norming for $Y$, consequently $\|S\|=$ $\sup _{\alpha}\left\|S^{*} y_{\alpha}^{*}\right\|$. Let us calculate the norm of $S .\|S\| \geqslant\left\|S^{*}\left(y_{\alpha_{0}}^{*}\right)\right\|=(1+\eta)\left\|z^{*}\right\|=1+\eta$.

On the other hand for $\alpha \neq \alpha_{0}$ we obtain

$$
\left\|S^{*}\left(y_{\alpha}^{*}\right)\right\| \leqslant 1+\rho\left(\left\|z^{*}-T^{*}\left(y_{\alpha_{0}}^{*}\right)\right\|+\eta\left\|z^{*}\right\|\right)<1+\rho(2 k+\eta)=1+\eta .
$$

Therefore,

$$
\|S\|=\left\|S^{*}\left(y_{\alpha_{0}}^{*}\right)\right\|=(1+\eta)\left\|z^{*}\right\|=\left|y_{\alpha_{0}}^{*}(S(z))\right| \leqslant\|S(z)\| \leqslant\|S\| .
$$

So, we have $\|S\|=\|S(z)\|=1+\eta$. Also, $\|S-T\| \leqslant \eta+\left\|z^{*}-T^{*}\left(y_{\alpha_{0}}^{*}\right)\right\|<\eta+2 k$.

Define $F:=\frac{S}{\|S\|}$. Then $\|F\|=\|F(z)\|=1$ and $\|S-F\|=\|S\|\left(1-\frac{1}{1+\eta}\right)=\eta$. So, $\|T-F\|<2 k+2 \eta$.

Therefore, we have that $\|z-x\|<\varepsilon / k$ and $\|T-F\|<2 k \frac{1+\rho}{1-\rho}$. obtain

Let us substitute $k=\sqrt{\frac{\varepsilon}{2} \cdot \frac{1-\rho}{1+\rho}}$ (here we need $\varepsilon \leqslant \frac{2(1-\rho)}{1+\rho}$ to have $k \in[\varepsilon / 2,1)$ ). Then we

$$
\max \{\|z-x\|,\|T-F\|\}<\sqrt{2 \varepsilon} \sqrt{\frac{1+\rho}{1-\rho}} .
$$

Finally, if $\varepsilon>\frac{2(1-\rho)}{1+\rho}$, we can use the triangle inequality to get the evident estimate $\max \{\| z-$ $x\|\| T-F \|,\} \leqslant 2$.

Our next goal is to give an improvement for a uniformly non-square domain space $X$. We recall that uniformly non-square spaces were introduced by James [9] as those spaces whose two-dimensional subspaces are uniformly separated (in the sense of Banach-Mazur distance) from $\ell_{1}^{(2)}$. A Banach space $X$ is uniformly non-square if and only if there is $\alpha>0$ such that

$$
\frac{1}{2}(\|x+y\|+\|x-y\|) \leqslant 2-\alpha
$$

for all $x, y \in B_{X}$. The parameter of uniform non-squareness of $X$, which we denote $\alpha(X)$, is the best possible value of $\alpha$ in the above inequality. In other words,

$$
\alpha(X):=2-\sup _{x, y \in B_{X}}\left\{\frac{1}{2}(\|x+y\|+\|x-y\|)\right\} .
$$

In [6, Theorem 3.3] it was proved that for a uniformly non-square space $X$ with the parameter of uniform non-squareness $\alpha(X)>\alpha_{0}>0$

$$
\Phi_{X}^{S}(\varepsilon) \leqslant \sqrt{2 \varepsilon} \sqrt{1-\frac{1}{3} \alpha_{0}} \text { for } \varepsilon \in\left(0, \frac{1}{2}-\frac{1}{6} \alpha_{0}\right) .
$$

To obtain this fact the authors proved the following technical result. 
Lemma 2. Let $X$ be a Banach space with $\alpha(X)>\alpha_{0}$. Then for every $x \in S_{X}, y \in X$ and every $k \in\left(0, \frac{1}{2}\right]$ if $\|x-y\| \leqslant k$ then

$$
\left\|x-\frac{y}{\|y\|}\right\| \leqslant 2 k\left(1-\frac{1}{3} \alpha_{0}\right) .
$$

Theorem 2. Let $X$ and $Y$ be Banach spaces such that $\beta(Y) \leqslant \rho, X$ is uniformly non-square with $\alpha(X)>\alpha_{0}$, and $\varepsilon_{0}=\min \left\{\frac{2}{\left(1-1 / 3 \alpha_{0}\right)} \frac{1-\rho}{1+\rho}, \frac{1}{2} \frac{1+\rho}{1-\rho}\left(1-1 / 3 \alpha_{0}\right)\right\}$. Then for any $0<\varepsilon<\varepsilon_{0}$

$$
\Phi^{S}(X, Y, \varepsilon) \leqslant \sqrt{2 \varepsilon\left(1-\frac{1}{3} \alpha_{0}\right)} \sqrt{\frac{1+\rho}{1-\rho}} .
$$

Before proving the theorem, we need a preliminary result.

Lemma 3. Let $X$ be a Banach space with $\alpha(X)>\alpha_{0}$. Then for every $0<\varepsilon<1$ and for every $\left(x, x^{*}\right) \in S_{X} \times B_{X^{*}}$ with $x^{*}(x)>1-\varepsilon$, and for every $k \in\left[\frac{\varepsilon}{2\left(1-1 / 3 \alpha_{0}\right)}\right.$, $\left.\frac{1}{2}\right]$ there is $\left(y, y^{*}\right) \in \Pi(X)$ such that

$$
\|x-y\|<\frac{\varepsilon}{k} \quad \text { and } \quad\left\|x^{*}-y^{*}\right\|<2 k\left(1-\frac{1}{3} \alpha_{0}\right) .
$$

Proof. The reasoning is almost the same as in Lemma 1. We have that $\frac{x^{*}}{\left\|x^{*}\right\|}(x)>1-\eta$ for $\eta=1-\frac{1-\varepsilon}{\left\|x^{*}\right\|}$ and we can apply Proposition 2 for every $\tilde{k} \in(0,1 / 2]$. Let us take

$$
\tilde{k}=\frac{k\left(\left\|x^{*}\right\|-(1-\varepsilon)\right)}{\varepsilon\left\|x^{*}\right\|} .
$$

The inequality $\left\|x^{*}\right\| \geqslant x^{*}(x)>1-\varepsilon$ implies that $\tilde{k}>0$. On the other hand, $\tilde{k}=k\left(\frac{1}{\varepsilon}-\frac{(1-\varepsilon)}{\varepsilon\left\|x^{*}\right\|}\right) \leqslant$ $k\left(\frac{1}{\varepsilon}-\frac{(1-\varepsilon)}{\varepsilon}\right)=k<1 / 2$, so for this $\tilde{k}$ we can find $\zeta^{*} \in X^{*}$ and $z \in S_{X}$ such that

$$
\zeta^{*}(z)=\left\|\zeta^{*}\right\|, \quad\|x-z\|<\frac{\eta}{\tilde{k}}, \quad\left\|\frac{x^{*}}{\left\|x^{*}\right\|}-\zeta^{*}\right\|<\tilde{k} .
$$

Consider $z^{*}=\frac{\zeta^{*}}{\left\|\zeta^{*}\right\|}$. According to Lemma 2

$$
\left\|\frac{x^{*}}{\left\|x^{*}\right\|}-z^{*}\right\|<2 \tilde{k}\left(1-\frac{1}{3} \alpha_{0}\right) .
$$

Then $\|x-z\|<\varepsilon / k$ and

$$
\begin{gathered}
\left\|x^{*}-z^{*}\right\|=\left\|x^{*}\right\| \cdot\left\|\frac{x^{*}}{\left\|x^{*}\right\|}-\frac{z^{*}}{\left\|x^{*}\right\|}\right\| \leqslant\left\|x^{*}\right\|\left(\left\|\frac{x^{*}}{\left\|x^{*}\right\|}-z^{*}\right\|+\left\|z^{*}-\frac{z^{*}}{\left\|x^{*}\right\|}\right\|\right)= \\
=\left\|x^{*}\right\|\left(2 \tilde{k}\left(1-1 / 3 \alpha_{0}\right)+\left|1-\frac{1}{\left\|x^{*}\right\|}\right|\right)= \\
=2 \frac{k\left(\left\|x^{*}\right\|-(1-\varepsilon)\right)}{\varepsilon}\left(1-1 / 3 \alpha_{0}\right)+1-\left\|x^{*}\right\| \leqslant 2 k\left(1-1 / 3 \alpha_{0}\right) .
\end{gathered}
$$

The last inequality holds, because if we consider the function

$$
f(t)=\frac{2 k\left(1-1 / 3 \alpha_{0}\right) \cdot(t-(1-\varepsilon))}{\varepsilon}+1-t
$$

with $t \in(1-\varepsilon, 1]$, then $f^{\prime} \geqslant 0$ if $k \geqslant \frac{\varepsilon}{2\left(1-1 / 3 \alpha_{0}\right)}$, so $\max f=f(1)=2 k\left(1-\frac{1}{3} \alpha_{0}\right)$. 
Proof of Theorem 2. The proof is a minor modification of the one given for Theorem 1.

In order to get (5) for $\varepsilon<\varepsilon_{0}$ we consider $T \in S_{L(X, Y)}$ and $x \in S_{X}$ such that $\|T(x)\|>1-\varepsilon$. Since $Y$ has the property $\beta$, there is $\alpha_{0} \in \Lambda$ such that $\left|y_{\alpha_{0}}^{*}(T(x))\right|>1-\varepsilon$. By Lemma 3, for any $k \in\left[\frac{\varepsilon}{2\left(1-1 / 3 \alpha_{0}\right)}, \frac{1}{2}\right]$ and for any $\varepsilon>0$ there exist $z^{*} \in S_{X^{*}}$ and $z \in S_{X}$ such that $\left|z^{*}(z)\right|=1,\|z-x\|<\varepsilon / k$ and $\left\|z^{*}-T^{*}\left(y_{\alpha_{0}}^{*}\right)\right\|<2 k\left(1-1 / 3 \alpha_{0}\right)$.

For $\eta=2 k\left(1-1 / 3 \alpha_{0}\right) \frac{\rho}{1-\rho}$ we define $S \in L(X, Y)$ by formula (4) and take $F:=\frac{S}{\|S\|}$. By the same argumentation as before, we have that

$$
\|x-z\|<\varepsilon / k \text { and }\|T-F\|<2 k\left(1-\frac{1}{3} \alpha_{0}\right) \frac{1+\rho}{1-\rho} .
$$

Let us substitute $k=\sqrt{\frac{\varepsilon}{2\left(1-1 / 3 \alpha_{0}\right)} \cdot \frac{1-\rho}{1+\rho}}$ (here we need $\varepsilon<\varepsilon_{0}$ ). Then we obtain that

$$
\max \{\|z-x\|,\|T-F\|\}<\sqrt{2 \varepsilon\left(1-\frac{1}{3} \alpha_{0}\right)} \sqrt{\frac{1+\rho}{1-\rho}} .
$$

\section{Estimation from below.}

3.1. Improvement for $\Phi\left(\ell_{1}^{(2)}, Y, \varepsilon\right)$. We tried our best, but unfortunately we could not find an example demonstrating the sharpness of (1) in Theorem 1. So, our goal is less ambitious. We are going to present examples of pairs $(X, Y)$ for which the estimation of $\Phi(X, Y, \varepsilon)$ from below is reasonably close to the estimation from above given in (1).

Theorem 2 shows that in order to check the sharpness of Theorem 1 one has to try those domain spaces $X$ that are not uniformly non-square. The simplest of them is $X=\ell_{1}^{(2)}$. In [5, Example 2.5] this space worked perfectly for the Bishop-Phelps-Bollobás modulus for functionals. Nevertheless, this is not so when one deals with the Bishop-Phelps-Bollobás modulus for operators. Namely, the following theorem demonstrates that for $X=\ell_{1}^{(2)}$ the estimation given in Theorem 1 can be improved.

Theorem 3. Let $Y$ be Banach spaces and $\beta(Y) \leqslant \rho$. Then

$$
\Phi^{S}\left(\ell_{1}^{(2)}, Y, \varepsilon\right) \leqslant \Phi\left(\ell_{1}^{(2)}, Y, \varepsilon\right) \leqslant \min \left\{\sqrt{2 \varepsilon} \frac{1+\rho}{\sqrt{1-\rho^{2}+\frac{\varepsilon}{2} \rho^{2}}+\rho \sqrt{\frac{\varepsilon}{2}}}, 1\right\} .
$$

To prove this theorem we need a preliminary result.

Lemma 4. Let $Y$ be a Banach space such that $\beta(Y) \leqslant \rho, y \in B_{Y},\left\{y_{\alpha}: \alpha \in \Lambda\right\} \subset S_{Y}$, $\left\{y_{\alpha}^{*}: \alpha \in \Lambda\right\} \subset S_{Y}^{*}$ be the sets from Definition 2. For given $r \in(0,1), \alpha_{0} \in \Lambda$ suppose that $y_{\alpha_{0}}^{*}(y) \geqslant 1-r$. Then there is $z \in S_{Y}$ such that

(i) $y_{\alpha_{0}}^{*}(z)=1$;

(ii) $\left|y_{\alpha}^{*}(z)\right| \leqslant 1$ for all $\alpha \in \Lambda$;

(iii) $\|y-z\| \leqslant \frac{r(1+\rho)}{1-\rho+\rho r}$.

Proof. Suppose that $y_{\alpha_{0}}^{*}(y)=1-r_{0}, r_{0} \in[0, r]$. According to (i) of Definition $2 y_{\alpha_{0}}^{*}\left(y_{\alpha_{0}}\right)=1$. Let us check the properties (i)-(iii) for

$$
z:=\frac{r_{0}}{1-\rho+\rho r_{0}} y_{\alpha_{0}}+\left(1-\frac{r_{0} \rho}{1-\rho+\rho r_{0}}\right) y
$$


(i) $y_{\alpha_{0}}^{*}(z)=\frac{r_{0}}{1-\rho+\rho r_{0}}+\left(1-\frac{r_{0} \rho}{1-\rho+\rho r_{0}}\right)\left(1-r_{0}\right)=1$;

(ii) For every $\alpha \neq \alpha_{0}$ we have $\left|y_{\alpha}^{*}(z)\right| \leqslant \frac{r_{0}}{1-\rho+\rho r_{0}} \cdot \rho+\left(1-\frac{r_{0} \rho}{1-\rho+\rho r_{0}}\right)=1$;

(iii) As $\left\{y_{\alpha}^{*}: \alpha \in \Lambda\right\} \subset S_{Y}^{*}$ is a 1-norming subset, so $\|y-z\|=\sup _{\alpha \in \Lambda}\left|y_{\alpha}^{*}(y-z)\right|$. Notice that $\left|y_{\alpha_{0}}^{*}(y-z)\right| \leqslant r$, and for every $\alpha \neq \alpha_{0}$ we have

$$
\left|y_{\alpha}^{*}(y-z)\right|=\left|\frac{r_{0}}{1-\rho+\rho r_{0}} y_{\alpha}^{*}(y)-\frac{r_{0}}{1-\rho+\rho r_{0}} y_{\alpha}^{*}\left(y_{\alpha_{0}}\right)\right| \leqslant \frac{r_{0}(1+\rho)}{1-\rho+\rho r_{0}} \leqslant \frac{r(1+\rho)}{1-\rho+\rho r} .
$$

So, $\|y-z\| \leqslant \max \left\{r, \frac{r(1+\rho)}{1-\rho+\rho r}\right\}=\frac{r(1+\rho)}{1-\rho+\rho r}$.

Finally, (i) and (ii) imply that $z \in S_{Y}$.

Remark 3. For every operator $T \in L\left(\ell_{1}^{(2)}, Y\right)$

$$
\|T\|=\max \left\{\left\|T\left(e_{1}\right)\right\|,\left\|T\left(e_{2}\right)\right\|\right\}
$$

Moreover, if the operator $T \in L\left(\ell_{1}^{(2)}, Y\right)$ attains its norm at some point $x \in S_{\ell_{1}^{(2)}}$ which does not coincide with $\pm e_{1}$ and $\pm e_{2}$, then either the segment $\left[T\left(e_{1}\right), T\left(e_{2}\right)\right]$, or $\left[T\left(e_{1}\right),-T\left(e_{2}\right)\right]$ has to lie on the sphere $\|T\| S_{Y}$.

Proof of Theorem 3. Let us denote $A(\rho, \varepsilon):=\sqrt{2 \varepsilon} \frac{1+\rho}{\sqrt{1-\rho^{2}+\frac{\varepsilon}{2} \rho^{2}}+\rho \sqrt{\frac{\varepsilon}{2}}}$. Notice that $A(\rho, \varepsilon)$ is increasing as a function of $\rho$, in particular $\sqrt{2 \varepsilon}=A(0, \varepsilon) \leqslant A(\rho, \varepsilon) \leqslant A(1, \varepsilon)=2$.

We are going to demonstrate that for every pair $(x, T) \in \Pi_{\varepsilon}\left(\ell_{1}^{(2)}, Y\right)$ there exists a pair $(y, F) \in \Pi\left(\ell_{1}^{(2)}, Y\right)$ with

$$
\max \{\|x-y\|,\|T-F\|\} \leqslant \min \{A(\rho, \varepsilon), 1\}
$$

Without loss of generality suppose that $x=(t(1-\delta), t \delta), \delta \in[0,1 / 2], t \in[1-\varepsilon, 1]$. Evidently, $\|x\|=t$. First, we make sure that $\Phi\left(\ell_{1}^{(2)}, Y, \varepsilon\right) \leqslant 1$. Indeed, we can always approximate $(x, T)$ by the pair $y:=e_{1}$ and $F$ determined by formula $F\left(e_{i}\right):=T\left(e_{i}\right) /\left\|T\left(e_{i}\right)\right\|$. Then $\left\|x-e_{1}\right\|=2 t \delta+1-t \leqslant 1$ and $\|T-F\| \leqslant 1$.

It remains to show that $\Phi\left(\ell_{1}^{(2)}, Y, \varepsilon\right) \leqslant A(\rho, \varepsilon)$, when $A(\rho, \varepsilon)<1$. As $A(\rho, \varepsilon) \geqslant \sqrt{2 \varepsilon}$ we must consider $\varepsilon \in(0,1 / 2)$. Since $Y$ has the property $\beta$, we can select an $\alpha_{0}$ such that $\left|y_{\alpha_{0}}^{*}(T(x))\right|>1-\varepsilon$. Without loss of generality we may assume $y_{\alpha_{0}}^{*}(T(x))>1-\varepsilon$. Then $y_{\alpha_{0}}^{*}\left(T\left(\frac{x}{t}\right)\right)>1-\varepsilon^{\prime}$, where $\varepsilon^{\prime}=\frac{\varepsilon-(1-t)}{t} \in(0, \varepsilon)$. Therefore,

$$
y_{\alpha_{0}}^{*}\left(T\left(e_{1}\right)\right)>1-\frac{\varepsilon^{\prime}}{1-\delta} \quad \text { and } \quad y_{\alpha_{0}}^{*}\left(T\left(e_{2}\right)\right)>1-\frac{\varepsilon^{\prime}}{\delta} \text {. }
$$

We are searching for an approximation of $(x, T)$ by a pair $(y, F) \in \Pi\left(\ell_{1}^{(2)}, Y\right)$. Let us consider two cases:

Case I: $2 t \delta+1-t \leqslant A(\rho, \varepsilon)$. In this case we approximate $(x, T)$ by the vector $y:=e_{1}$ and the operator $F$ such that $F\left(e_{1}\right):=\frac{T\left(e_{1}\right)}{\left\|T\left(e_{1}\right)\right\|}, F\left(e_{2}\right):=T\left(e_{2}\right)$. Then

$$
\|x-y\| \leqslant 2 t \delta+1-t \leqslant A(\rho, \varepsilon) \text { and }\|T-F\| \leqslant 1-\left\|T\left(e_{1}\right)\right\| \leqslant \frac{\varepsilon}{1-\delta} \leqslant 2 \varepsilon \leqslant A(\rho, \varepsilon) .
$$


Case II: $2 t \delta+1-t>A(\rho, \varepsilon)$. Remark, that in this case $2 t \delta+1-t>\sqrt{2 \varepsilon}$, and consequently (here we use that $A(\rho, \varepsilon) \geqslant \sqrt{2 \varepsilon}, \varepsilon \in(0,1 / 2)$ and $t \in(0,1])$,

$$
\delta>\frac{\sqrt{2 \varepsilon}-(1-t)}{2 t} \geqslant \varepsilon^{\prime}
$$

According to (7) we can apply Lemma 4 for the points $T\left(e_{1}\right)$ and $T\left(e_{2}\right)$ with $r=\frac{\varepsilon^{\prime}}{\delta}<1$. So, there are $z_{1}, z_{2} \in S_{Y}$ such that $y_{\alpha_{0}}^{*}\left(z_{1}\right)=y_{\alpha_{0}}^{*}\left(z_{2}\right)=1$ and

$$
\max \left\{\left\|T\left(e_{1}\right)-z_{1}\right\|,\left\|T\left(e_{2}\right)-z_{2}\right\|\right\} \leqslant \frac{\frac{\varepsilon^{\prime}}{\delta}(1+\rho)}{1-\rho+\rho \frac{\varepsilon^{\prime}}{\delta}} .
$$

Denote $y:=x / t \in S_{\ell_{1}^{(2)}}$ and define $F$ as follows

$$
F\left(e_{1}\right):=z_{1}, \quad F\left(e_{2}\right):=z_{2} .
$$

Then $\|F\|=1,\|F(y)\| \geqslant y_{\alpha_{0}}^{*}(F y)=1$, so $F$ attains its norm at $y$ and

$$
\|T-F\| \leqslant \frac{\frac{\varepsilon^{\prime}}{\delta}(1+\rho)}{1-\rho+\rho \frac{\varepsilon^{\prime}}{\delta}} .
$$

So, in this case

$$
\|x-y\| \leqslant \varepsilon \leqslant A(\rho, \varepsilon) \text { and }\|T-F\| \leqslant \frac{(1+\rho) \frac{\varepsilon-1+t}{t \delta}}{1-\rho+\rho \frac{\varepsilon-1+t}{t \delta}} \text {. }
$$

To prove our statement we must show that if $2 t \delta+1-t>A(\rho, \varepsilon)$, then $\frac{(1+\rho) \frac{\varepsilon-1+t}{t \delta}}{1-\rho+\rho \frac{\varepsilon-1+t}{t \delta}} \leqslant$ $A(\rho, \varepsilon)$. Let us denote $f(t, \delta)=2 t \delta+1-t$ and $g(t, \delta)=\frac{(1+\rho) \frac{\varepsilon-1+t}{t \delta}}{1-\rho+\rho \frac{\varepsilon-1+t}{t \delta}}=\frac{(1+\rho)(\varepsilon-1+t)}{(1-\rho) t \delta+\rho(\varepsilon-1+t)}$. So, we need to demonstrate that

$$
\min \{f(t, \delta), g(t, \delta)\} \leqslant A(\rho, \varepsilon) \text { for all } \delta \in[0,1 / 2] \text { and for all } t \in[1-\varepsilon, 1] \text {. }
$$

Notice that for every fixed $t \in[1-\varepsilon, 1]$ the function $f(t, \delta)$ is increasing as $\delta$ increases and $g(t, \delta)$ is decreasing as $\delta$ increases. So, if we find $\delta_{0}$ such that $f(t, \delta)=g(t, \delta)$, then $\min \{f(t, \delta), g(t, \delta)\} \leqslant f\left(t, \delta_{0}\right)$. If we denote $u=f(t, \delta)=2 t \delta+1-t$ then the equation $f(t, \delta)=g(t, \delta)$ transforms to

$$
u=2-\frac{2(1-\rho)(u-\varepsilon)}{(t-1+\varepsilon)(1+\rho)+(u-\varepsilon)(1-\rho)} .
$$

The right-hand side of this equation is increasing as $t$ increases, so the positive solution of equation (9) $u_{t}$ is also increasing. This means that we obtain the greatest possible solution, if we substitute $t=1$. Then we get the equation

$$
u^{2} \frac{1+\rho}{2}+u \rho \varepsilon-\varepsilon(1+\rho)=0 .
$$

From here $u=A(\rho, \varepsilon)$, and so, inequality (8) holds. 
3.2. Estimation from below for $\Phi^{S}\left(\ell_{1}^{(2)}, Y, \varepsilon\right)$. So, if $X=\ell_{1}^{(2)}$, the estimation for the Bishop-Phelps-Bollobás modulus is somehow better than in Theorem 1. Nevertheless, considering $\ell_{1}^{(2)}$ we can obtain some interesting estimations from below for $\Phi^{S}\left(\ell_{1}^{(2)}, Y, \varepsilon\right)$. Notice that estimations (1) and (6) give the same asymptotic behaviour when $\varepsilon$ tends to 0. Our next proposition gives an estimation for $\Phi^{S}\left(\ell_{1}^{(2)}, Y, \varepsilon\right)$ from below, when $\beta(Y)=0$.

Theorem 4. For every Banach space $Y$

$$
\Phi^{S}\left(\ell_{1}^{(2)}, Y, \varepsilon\right) \geqslant \min \{\sqrt{2 \varepsilon}, 1\} .
$$

In particular, $\Phi^{S}\left(\ell_{1}^{(2)}, Y, \varepsilon\right)=\min \{\sqrt{2 \varepsilon}, 1\}$ if $\beta(Y)=0$.

Proof. To prove our statement we must show that $\Phi^{S}\left(\ell_{1}^{(2)}, Y, \varepsilon\right) \geqslant \sqrt{2 \varepsilon}$ for $\varepsilon \in(0,1 / 2)$. The remaining inequality $\Phi^{S}\left(\ell_{1}^{(2)}, Y, \varepsilon\right) \geqslant 1$ for $\varepsilon>1 / 2$ will follow from the monotonicity of $\Phi^{S}\left(\ell_{1}^{(2)}, Y, \cdot\right)$. So, for every $\varepsilon \in(0,1 / 2)$ and for every $\delta>0$ we are looking for a pair $(x, T) \in \Pi_{\varepsilon}^{S}\left(\ell_{1}^{(2)}, Y\right)$ such that $\max \{\|x-y\|,\|T-F\|\} \geqslant \sqrt{2 \varepsilon}-\delta$. for every pair $(y, F) \in$ $\Pi\left(\ell_{1}^{(2)}, Y\right)$. Fix $\xi \in S_{Y}$ and $\varepsilon_{0}<\varepsilon$ such that $\sqrt{2 \varepsilon_{0}}>\sqrt{2 \varepsilon}-\delta$. Consider the following operator $T \in S_{L\left(\ell_{1}^{(2)}, Y\right)}:$

$$
T\left(z_{1}, z_{2}\right)=\left(z_{1}+\left(1-\sqrt{2 \varepsilon_{0}}\right) z_{2}\right) \xi
$$

and take $x=\left(1-\sqrt{\varepsilon_{0} / 2}, \sqrt{\varepsilon_{0} / 2}\right) \in S_{\ell_{1}^{(2)}}$. Then $\|T(x)\|=1-\varepsilon_{0}>1-\varepsilon$. To approximate the pair $(x, T)$ by a pair $(y, F) \in \Pi\left(\ell_{1}^{(2)}, Y\right)$ we have two possibilities: either $y$ is an extreme point of $B_{\ell_{1}^{(2)}}$ or $F$ attains its norm at a point that belongs to $\operatorname{conv}\left\{e_{1}, e_{2}\right\}$, and so attains its norm at both points $e_{1}, e_{2}$. In the first case we are forced to have $y=(1,0)$, and then $\|x-y\|=\sqrt{2 \varepsilon_{0}}>\sqrt{2 \varepsilon}-\delta$. In the second case we have $\|F-T\| \geqslant\left\|F\left(e_{2}\right)-T\left(e_{2}\right)\right\| \geqslant$ $\left\|F\left(e_{2}\right)\right\|-\left\|T\left(e_{2}\right)\right\|=\sqrt{2 \varepsilon_{0}}>\sqrt{2 \varepsilon}-\delta$.

Our next goal is to estimate the spherical Bishop-Phelps-Bollobás modulus from below for the values of parameter $\rho$ between $1 / 2$ and 1 . Fix $\rho \in\left[\frac{1}{2}, 1\right)$ and denote $Y_{\rho}$ the linear space $\mathbb{R}^{2}$ equipped with the norm

$$
\|x\|_{\rho}=\max \left\{\left|x_{1}+\left(2-\frac{1}{\rho}\right) x_{2}\right|,\left|x_{2}+\left(2-\frac{1}{\rho}\right) x_{1}\right|,\left|x_{1}-x_{2}\right|\right\} .
$$

In other words,

$$
\left\|\left(x_{1}, x_{2}\right)\right\|= \begin{cases}\left|x_{1}-x_{2}\right|, & \text { if } x_{1} x_{2} \leqslant 0 \\ \left|x_{1}+\left(2-\frac{1}{\rho}\right) x_{2}\right|, & \text { if } x_{1} x_{2}>0 \text { and }\left|x_{1}\right|>\left|x_{2}\right| ; \\ \left|x_{2}+\left(2-\frac{1}{\rho}\right) x_{1}\right|, & \text { if } x_{1} x_{2}>0 \text { and }\left|x_{1}\right| \leqslant\left|x_{2}\right| .\end{cases}
$$

and the unit ball $B_{\rho}$ of $X_{\rho}$ is the hexagon absdef, where $a=(1,0) ; b=\left(\frac{\rho}{3 \rho-1}, \frac{\rho}{3 \rho-1}\right) ; c=$ $(0,1) ; d=(-1,0) ; e=\left(-\frac{\rho}{3 \rho-1}, \frac{\rho}{3 \rho-1}\right)$; and $f=(0,-1)$.

The dual space to $Y_{\rho}$ is $\mathbb{R}^{2}$ equipped with the polar to $B_{\rho}$ as its unit ball. So, the norm on $Y^{*}=Y_{\rho}^{*}$ is given by the formula

$$
\|x\|_{\rho}^{*}=\left\|\left(x_{1}, x_{2}\right)\right\|^{*}=\max \left\{\left|x_{1}\right|,\left|x_{2}\right|, \frac{\rho}{3 \rho-1}\left|x_{1}+x_{2}\right|\right\},
$$




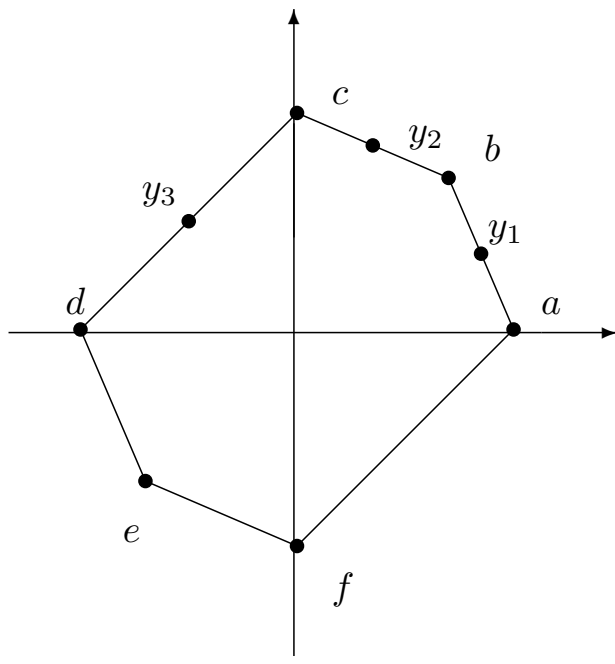

Figure 1

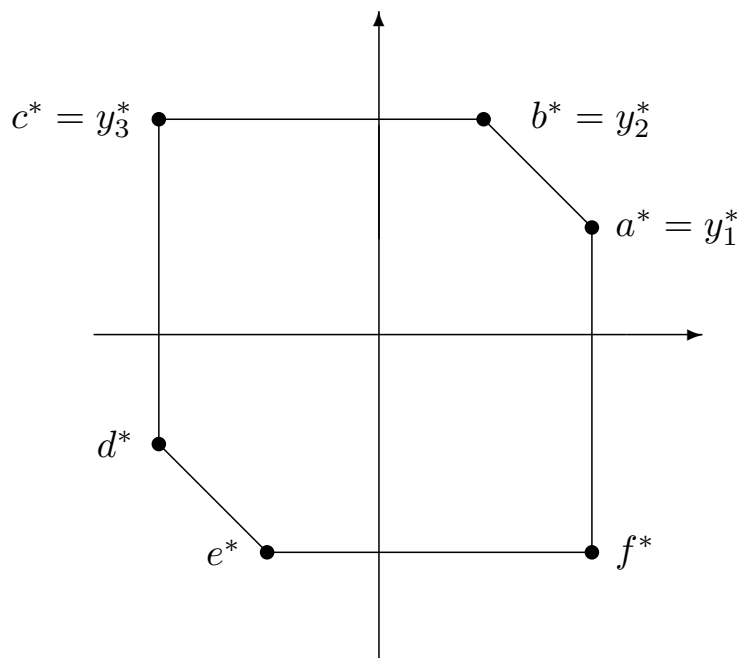

Figure 2

and the unit ball $B_{\rho}^{*}$ of $Y_{\rho}^{*}$ is the hexagon $a^{*} b^{*} c^{*} d^{*} e^{*} f^{*}$, where $a^{*}=\left(1,2-\frac{1}{\rho}\right) ; b^{*}=$ $\left(2-\frac{1}{\rho}, 1\right) ; c^{*}=(-1,1) ; d^{*}=\left(-1,-\left(2-\frac{1}{\rho}\right)\right) ; e^{*}=\left(-\left(2-\frac{1}{\rho}\right),-1\right) ;$ and $f^{*}=(1,-1)$. The corresponding spheres $S_{\rho}$ and $S_{\rho}^{*}$ are shown on Figures 1 and 2 respectively.

Proposition 3. In the space $Y=Y_{\rho}$

$$
\beta(Y) \leqslant \rho .
$$

Proof. Consider two sets:

$$
\left\{y_{1}=\left(\frac{2 \rho^{2}}{3 \rho-1}, \frac{\rho-\rho^{2}}{3 \rho-1}\right), y_{2}=\left(\frac{\rho-\rho^{2}}{3 \rho-1}, \frac{2 \rho^{2}}{3 \rho-1}\right), y_{3}=\left(-\frac{1}{2}, \frac{1}{2}\right)\right\} \subset S_{Y_{\rho}}
$$

and $\left\{y_{1}^{*}=\left(1,2-\frac{1}{\rho}\right), y_{2}^{*}=\left(2-\frac{1}{\rho}, 1\right), y_{3}^{*}=(-1,1)\right\} \subset S_{Y_{\rho}^{*}}$.

Then $\|y\|=\sup \left\{\left|y_{n}^{*}(y)\right|: n=1,2,3\right\}$ for all $y \in Y_{\rho}, y_{n}^{*}\left(y_{n}\right)=1$ for $n=1,2,3$ and $\left|y_{i}^{*}\left(y_{j}\right)\right| \leqslant \rho$ for all $i \neq j$. Indeed, $y_{1}^{*}\left(y_{1}\right)=\frac{2 \rho^{2}+2 \rho-2 \rho^{2}-1+\rho}{3 \rho-1}=1 ; \quad y_{1}^{*}\left(y_{2}\right)=\frac{\rho-\rho^{2}+4 \rho^{2}-2 \rho}{3 \rho-1}=\rho$; $y_{1}^{*}\left(y_{3}\right)=\frac{-1}{2}+1-\frac{1}{2 \rho}=-\frac{1-\rho}{2 \rho} \geqslant-\rho$, consequently $\left|y_{1}^{*}\left(y_{3}\right)\right| \leqslant \rho$ (here appears the restriction $\rho \geqslant 1 / 2) ; \quad y_{2}^{*}\left(y_{1}\right)=y_{1}^{*}\left(y_{2}\right)=\rho ; \quad y_{2}^{*}\left(y_{2}\right)=y_{1}^{*}\left(y_{1}\right)=1 ; \quad y_{2}^{*}\left(y_{3}\right)=-y_{1}^{*}\left(y_{3}\right) \leqslant \rho ; \quad\left|y_{3}^{*}\left(y_{1}\right)\right|=$ $\left|\frac{-2 \rho^{2}+\rho-\rho^{2}}{3 \rho-1}\right|=\rho ; y_{3}^{*}\left(y_{2}\right)=-y_{3}^{*}\left(y_{1}\right)=\rho$; and finally $y_{3}^{*}\left(y_{3}\right)=\frac{1}{2}+\frac{1}{2}=1$.

Theorem 5. Let $\rho \in[1 / 2,1), 0<\varepsilon<1$. Then, for the space $Y=Y_{\rho}$ one has

$$
\Phi^{S}\left(\ell_{1}^{(2)}, Y, \varepsilon\right) \geqslant \min \left\{\sqrt{\frac{2 \rho \varepsilon}{1-\rho}}, 1\right\} .
$$

Proof. To prove our statement we must show that $\Phi^{S}\left(\ell_{1}^{(2)}, Y, \varepsilon\right) \geqslant \sqrt{\frac{2 \rho \varepsilon}{1-\rho}}$ for $\varepsilon \in\left(0, \frac{1-\rho}{2 \rho}\right)$. The remaining inequality $\Phi^{S}\left(\ell_{1}^{(2)}, Y, \varepsilon\right) \geqslant 1$ for $\varepsilon \geqslant \frac{1-\rho}{2 \rho}$ will follow from the monotonicity 
of $\Phi^{S}\left(\ell_{1}^{(2)}, Y, \cdot\right)$. So, for every $\varepsilon \in\left(0, \frac{1-\rho}{2 \rho}\right)$ and for every $\delta>0$ we are looking for a pair $(x, T) \in \Pi_{\varepsilon}^{S}\left(\ell_{1}^{(2)}, Y\right)$ such that

$$
\max \{\|x-y\|,\|T-F\|\} \geqslant \sqrt{\frac{2 \rho \varepsilon}{1-\rho}}-\delta
$$

for every pair $(y, F) \in \Pi\left(\ell_{1}^{(2)}, Y\right)$.

Fix an $\varepsilon_{0}<\varepsilon$ such that $\sqrt{\frac{2 \rho \varepsilon_{0}}{1-\rho}}>\sqrt{\frac{2 \rho \varepsilon}{1-\rho}}-\delta$. Consider the point

$$
x=\left(1-\frac{\sqrt{2 \rho \varepsilon_{0}}}{2 \sqrt{1-\rho}}, \frac{\sqrt{2 \rho \varepsilon_{0}}}{2 \sqrt{1-\rho}}\right) \in S_{\ell_{1}^{(2)}}
$$

and $T \in L\left(\ell_{1}^{(2)}, Y\right)$ such that

$$
T\left(e_{i}\right)=\sqrt{\frac{2 \rho \varepsilon_{0}}{1-\rho}} e_{i}+\left(1-\sqrt{\frac{2 \rho \varepsilon_{0}}{1-\rho}}\right) \cdot b,
$$

where $b=\left(\frac{\rho}{3 \rho-1}, \frac{\rho}{3 \rho-1}\right)$ is the extreme point of $S_{Y}$ from Figure 1. Notice that $\|T\|=\left\|T\left(e_{1}\right)\right\|=$ $\left\|T\left(e_{2}\right)\right\|=1$ and $\|T(x)\|=1-\varepsilon_{0}>1-\varepsilon$.

The part of $S_{\ell_{1}^{(2)}}$ consisting of points that have a distance to $x$ less than or equal to $\sqrt{\frac{2 \rho \varepsilon_{0}}{1-\rho}}$ lies on the segment $\left[e_{1}, e_{2}\right)$. Consequently, in order to approximate the pair $(x, T)$ we have two options: to approximate the point $x$ by $e_{1}$, and then we can take $F:=T$; or as $F$ choose an operator attaining its norm at some point of $\left(e_{1}, e_{2}\right)$ (and hence at all points of $\left[e_{1}, e_{2}\right]$ ), and then we can take $y:=x$.

In the first case we have $\|T-F\|=0$ and $\|x-y\|=\sqrt{\frac{2 \rho \varepsilon_{0}}{1-\rho}}>\sqrt{\frac{2 \rho \varepsilon}{1-\rho}}-\delta$. In the second case let us demonstrate that

$$
\|T-F\|=\max _{i}\left\|T\left(e_{i}\right)-F\left(e_{i}\right)\right\| \geqslant \sqrt{\frac{2 \rho \varepsilon_{0}}{1-\rho}} .
$$

If it is not so, then for both values of $i=1,2$

$$
\left\|T\left(e_{i}\right)-F\left(e_{i}\right)\right\|<\sqrt{\frac{2 \rho \varepsilon_{0}}{1-\rho}}=\left\|T\left(e_{i}\right)-b\right\| .
$$

Since $F$ attains its norm at all points of $\left[e_{1}, e_{2}\right]$, the line segment $F\left(\left[e_{1}, e_{2}\right]\right)$ should lie on a line segment of $S_{Y}$, but the previous inequality makes this impossible, because $T\left(e_{1}\right)$ and $T\left(e_{2}\right)$ lie on different line segments of $S_{Y}$ with $b$ being their only common point.

3.3. Non-continuity of the Bishop-Phelps-Bollobás modulus for operators. It is known [7, Theorem 3.3] that both (usual and spherical) Bishop-Phelps-Bollobás moduli for functionals are continuous with respect to $X$. As a consequence of Theorem 5 we will obtain that the Bishop-Phelps-Bollobás moduli of a pair $(X, Y)$ as a function of $Y$ are not continuous in the sense of Banach-Mazur distance.

Let $X$ and $Y$ be isomorphic. Recall that the Banach-Mazur distance between $X$ and $Y$ is the following quantity

$$
d(X, Y)=\inf \left\{\|T\|\left\|T^{-1}\right\|: \quad T: X \rightarrow Y \text { isomorphism. }\right\}
$$


A sequence $Z_{n}$ of Banach spaces is said to be convergent to a Banach space $Z$ if $d\left(Z_{n}, Z\right) \underset{n \rightarrow \infty}{\longrightarrow} 1$.

Notice, that $Y_{\rho} \underset{\rho \rightarrow 1}{\longrightarrow} \ell_{1}^{(2)}$.

Theorem 6. Let $\rho \in[1 / 2,1)$ and $Y_{\rho}$ be the spaces defined by $(10)$. Then for every $\varepsilon \in\left(0, \frac{1}{2}\right)$

$$
\Phi\left(\ell_{1}^{(2)}, Y_{\rho}, \varepsilon\right) \underset{\rho \rightarrow 1}{\longrightarrow} \Phi\left(\ell_{1}^{(2)}, \ell_{1}^{(2)}, \varepsilon\right) \text {, and } \Phi^{S}\left(\ell_{1}^{(2)}, Y_{\rho}, \varepsilon\right) \underset{\rho \rightarrow 1}{\longrightarrow} \Phi^{S}\left(\ell_{1}^{(2)}, \ell_{1}^{(2)}, \varepsilon\right) .
$$

Proof. On the one hand, from the Theorem 1 with $\rho=0$ we get for $\varepsilon \in\left(0, \frac{1}{2}\right)$

$$
\Phi^{S}\left(\ell_{1}^{(2)}, \ell_{1}^{(2)}, \varepsilon\right) \leqslant \Phi\left(\ell_{1}^{(2)}, \ell_{1}^{(2)}, \varepsilon\right) \leqslant \sqrt{2 \varepsilon}<1 .
$$

On the other hand, Theorem 5 gives

$$
\Phi\left(\ell_{1}^{(2)}, Y_{\rho}, \varepsilon\right) \geqslant \Phi^{S}\left(\ell_{1}^{(2)}, Y_{\rho}, \varepsilon\right) \geqslant \min \left\{\sqrt{\frac{2 \rho \varepsilon}{1-\rho}}, 1\right\} \underset{\rho \rightarrow 1}{\longrightarrow} 1 .
$$

3.4. Behavior of $\Phi^{S}(X, Y, \varepsilon)$ when $\varepsilon \rightarrow 0$. In subsection using two-dimensional spaces $Y$ we were able to give the estimation only for $\rho \in[1 / 2,1)$. This is not surprising, because in every $n$-dimensional Banach space with the property $\beta$ we have either $\rho=0$, or $\rho \geqslant \frac{1}{n}$. We did not find any mentioning of this in literature, so we give the proof of this fact.

Proposition 4. Let $Y^{(n)}$ be a Banach space of dimension $n$ with $\beta\left(Y^{(n)}\right) \leqslant \rho<\frac{1}{n}$. Then $Y^{(n)}$ is isometric to $\ell_{\infty}^{(n)}$, i.e. $\beta\left(Y^{(n)}\right)=0$.

We need one preliminary result.

Lemma 5. Let $Y^{(n)}$ be a Banach space of dimension $n$ with $\beta\left(Y^{(n)}\right) \leqslant \rho<\frac{1}{n}$ and $\left\{y_{\alpha}: \alpha \in\right.$ $\Lambda\} \subset S_{Y},\left\{y_{\alpha}^{*}: \alpha \in \Lambda\right\} \subset S_{Y}^{*}$ be the sets from Definition 2. Then $|\Lambda|=n$.

Proof. $|\Lambda| \geqslant n$, because $\left\{y_{\alpha}^{*}: \alpha \in \Lambda\right\}$ is a 1-norming subset. Assume that $|\Lambda|>n$. We are going to demonstrate that every subset of $\left\{y_{\alpha}: \alpha \in \Lambda\right\}$ consisting of $n+1$ elements is linearly independent.

Without loss of generality we can take a subset $\left\{y_{i}\right\}_{i=1}^{n+1} \subset\left\{y_{\alpha}: \alpha \in \Lambda\right\}$. Consider the corresponding linear combination $\sum_{i=1}^{n+1} a_{i} y_{i}$ with $\max \left|a_{i}\right|=1$ and let us check that $\sum_{i=1}^{n+1} a_{i} y_{i} \neq 0$. Let $j \leqslant n+1$ be a number such that $\left|a_{j}\right|=1$. Then we can estimate

$$
\left\|\sum_{i=1}^{n+1} a_{i} y_{i}\right\| \geqslant\left|y_{j}^{*}\left(\sum_{i=1}^{n+1} a_{i} y_{i}\right)\right|=\left|a_{j} y_{j}^{*}\left(y_{j}\right)+\sum_{\substack{i=1 \\ i \neq j}}^{n+1} a_{i} y_{j}^{*}\left(y_{i}\right)\right| \geqslant 1-\sum_{\substack{i=1 \\ i \neq j}}^{n+1}\left|a_{i}\right| \rho>0 .
$$

It follows that $Y^{(n)}$ contains $n+1$ linearly independent elements. This contradiction completes the proof.

Proof of Proposition 4. According to Definition 2 together with Lemma 5 there are two sets $\left\{y_{i}\right\}_{i=1}^{n} \subset S_{Y^{(n)}},\left\{y_{i}^{*}\right\}_{i=1}^{n} \subset S_{Y^{(n)}}^{*}$ such that $y_{i}^{*}\left(y_{i}\right)=1,\left|y_{i}^{*}\left(y_{j}\right)\right|<1 / n$ if $i \neq j,\|y\|=$ $\sup \left\{\left|y_{i}^{*}(y)\right|: i=1, \ldots, n\right\}$, for all $y \in Y$.

Let us define the operator $U: Y^{(n)} \rightarrow \ell_{\infty}^{(n)}$ by the formula $U(y):=\left(y_{1}^{*}(y), y_{2}^{*}(y), \ldots, y_{n}^{*}(y)\right)$. Obviously, $\|U(y)\|=\|y\|$ for all $y \in Y^{(n)}$, so, $U$ is isometry. Since $\operatorname{dim} Y^{(n)}=\operatorname{dim} \ell_{\infty}^{(n)}$, the operator $U$ is bijective. This means that $Y^{(n)}$ is isometric to $\ell_{\infty}^{(n)}$, and since $\beta\left(\ell_{\infty}^{(n)}\right)=0$, we have that $\beta\left(Y^{(n)}\right)=0$. 
So, in order to obtain all possible values of parameter $\rho$ we must consider spaces of higher dimensions. For every fixed dimension $n$ fix a $\rho \in\left[\frac{1}{n}, 1\right)$ and denote $Z=Z_{\rho}^{(n)}$ the linear space $\mathbb{R}^{n}$ equipped with the norm

$$
\|x\|=\max \left\{\left|x_{1}\right|,\left|x_{2}\right|, \ldots,\left|x_{n}\right|, \frac{1}{\rho n}\left|\sum_{i=1}^{n} x_{i}\right|\right\} .
$$

Proposition 5. Let $Z=Z_{\rho}^{(n)}$ with $n \geqslant 2$ and $\rho \in\left[\frac{1}{n}, 1\right)$. Then

$$
\beta(Z) \leqslant \rho .
$$

Proof. Consider two sets:

$$
\begin{gathered}
\left\{y_{j}=-\frac{1}{n-1+\rho n} \sum_{\substack{i=1 \\
i \neq j}}^{n} e_{i}+e_{j}, \quad z=\rho \sum_{i=1}^{n} e_{i}\right\} \subset S_{Z} \\
\left\{y_{j}^{*}=e_{j}, z^{*}=\frac{1}{\rho n} \sum_{i=1}^{n} e_{i}\right\} \subset S_{Z^{*}} .
\end{gathered}
$$

It follows directly from (11) that the subset $\left\{\left\{y_{j}^{*}\right\}_{i=1}^{n}, z^{*}\right\}$ is 1-norming. Also,

$$
y_{i}^{*}\left(y_{i}\right)=1,\left|y_{j}^{*}\left(y_{i}\right)\right|=\left|-\frac{1}{n-1+\rho n}\right| \leqslant \rho, y_{j}^{*}(z)=\rho, z^{*}(z)=1, z^{*}\left(y_{i}\right)=\frac{1}{n-1+\rho n} \leqslant \rho .
$$

Remark that in all our estimations of $\Phi^{S}(X, Y, \varepsilon)$ appears the multiplier $\sqrt{2 \varepsilon}$. So, in order to measure the behavior of $\Phi^{S}(X, Y, \varepsilon)$ at 0 , it is natural to introduce the following quantity

$$
\Psi(X, Y):=\limsup _{\varepsilon \rightarrow 0} \frac{\Phi^{S}(X, Y, \varepsilon)}{\sqrt{2 \varepsilon}} .
$$

Also define

$$
\Psi(\rho):=\sup _{Y: \beta(Y)=\rho} \sup _{X} \limsup _{\varepsilon \rightarrow 0} \Psi(X, Y)
$$

which measures the worst possible behavior in 0 of $\Phi^{S}(X, Y, \varepsilon)$ when $\beta(Y) \leqslant \rho$. From Theorem 1 we know that $\Psi(\rho) \leqslant \sqrt{\frac{1+\rho}{1-\rho}}$. Now we will estimate $\Psi(\rho)$ from below.

\section{Theorem 7.}

$$
\Psi(\rho) \geqslant \min \left\{\sqrt{\frac{2 \rho}{1-\rho}}, 1\right\}
$$

for all values of $\rho \in(0,1)$. 
Proof. From Theorem 4 we know that $\Psi(\rho) \geqslant 1$. So, we have to check that $\Psi(\rho) \geqslant \sqrt{\frac{2 \rho}{1-\rho}}$. In order to estimate $\Psi(\rho)$ from below for small $\varepsilon$ we consider the couple of spaces $\left(\ell_{1}^{(2)}, Z_{\rho}^{(n)}\right)$. Denote $z^{*}=\frac{1}{\rho n} \sum_{i=1}^{n} e_{i}$ and $\Gamma=\left\{x \in S_{Z}: z^{*}(x)=1\right\}$. Consider the point $x=(1-\delta, \delta)$ and the operator $T$ such that

$$
T\left(e_{1}\right)=\rho \sum_{i=1}^{n} e_{i} \text { and } T\left(e_{2}\right)=t \sum_{i=1}^{k} e_{i}+\sum_{i=k+1}^{n} e_{i},
$$

with $k=\frac{1}{2} n(1-\rho)+1+\theta \in \mathbb{N}$ being the nearest integer to $\frac{1}{2} n(1-\rho)+1($ so, $|\theta| \leqslant 1 / 2)$ and

$$
t=-1+\frac{4+4 \theta-2 n \rho \frac{\varepsilon_{0}}{\delta}}{n-n \rho+2+2 \theta}
$$

where $\delta>0$ will be defined later and $\varepsilon_{0}<\varepsilon$. Then $z^{*}(T(x))=1-\varepsilon_{0}>1-\varepsilon$, so $(x, T) \in$ $\Pi_{\varepsilon}^{S}\left(\ell_{1}^{(2)}, Z_{\rho}^{(n)}\right)$. Now we are searching for the best approximation of $(x, T)$ by a pair $(y, F) \in$ $\Pi\left(\ell_{1}^{(2)}, Z_{\rho}^{(n)}\right)$. As usual, we have two options:

I. We can approximate the point $x$ by $e_{1}$ and then we can take $F=T$. In this case we get

$$
\|x-y\|=2 \delta
$$

II. We can choose $F$ which attains its norm at all points of the segment $\left[e_{1}, e_{2}\right]$, and then we can take $y=x$. In this case $F\left(e_{1}\right)$ and $F\left(e_{2}\right)$ must lie in the same face. Besides, if $F\left(e_{1}\right) \notin \Gamma$, we have $\left\|T\left(e_{1}\right)-F\left(e_{1}\right)\right\|=1-\rho>\sqrt{2 \varepsilon} \sqrt{\frac{2 \rho}{1-\rho}}$ for $\varepsilon$ sufficiently small. To obtain better estimation we must have $F\left(e_{1}\right) \in \Gamma$ and, so, $F\left(e_{2}\right) \in \Gamma$. Then

$$
\|T-F\| \geqslant\left\|T\left(e_{2}\right)-F\left(e_{2}\right)\right\| \geqslant \inf _{h \in \Gamma}\left\|T\left(e_{2}\right)-h\right\| .
$$

Let us estimate the distance from $T\left(e_{2}\right)$ to the face $\Gamma$.

If $h=\sum_{i=1}^{n} h_{i} \in \Gamma$, then $\left|h_{i}\right| \leqslant 1$ and $z^{*}(h)=\frac{1}{\rho n} \sum_{i=1}^{n} h_{i}=1$. So, $\sum_{i=1}^{k} h_{i} \geqslant \rho n-(n-k)$, and

$$
\max h_{i} \geqslant \frac{1}{k}(\rho n-(n-k))=-1+\frac{4+4 \theta}{n(1-\rho)+2+2 \theta} .
$$

Therefore,

$$
\left\|T\left(e_{2}\right)-h\right\| \geqslant \max _{1 \leqslant i \leqslant k}\left|t-h_{i}\right| \geqslant\left|t-\max h_{i}\right|=\frac{2 n \rho \frac{\varepsilon_{0}}{\delta}}{n(1-\rho)+2+2 \theta} .
$$

Now let us define $\delta$ to be the positive solution of the equation $2 \delta=\frac{2 n \rho \frac{\varepsilon_{0}}{\delta}}{n(1-\rho)+2+2 \theta}$. Then $\delta=\frac{1}{2} \sqrt{2 \varepsilon_{0}} \sqrt{\frac{2 \rho}{1-\rho+(2+\theta) / n}}$. Denote $C(\varepsilon, \rho, n, \theta)=\sqrt{2 \varepsilon} \sqrt{\frac{2 \rho}{1-\rho+(2+\theta) / n}}$ and $C_{0}=C\left(\varepsilon_{0}, \rho, n, \theta\right)$. So, with this $\delta$ estimation (13) gives us $\|x-y\|=2 \delta=C_{0}$, and estimation (14) gives us

$$
\|T-F\| \geqslant \frac{2 n \rho \frac{\varepsilon_{0}}{\delta}}{n(1-\rho)+2+2 \theta}=C_{0} .
$$


In that way, we have shown that $\Phi^{S}\left(\ell_{1}^{(2)}, Z_{\rho}^{(n)}, \varepsilon\right) \geqslant C_{0}$. As $\varepsilon_{0}$ can be chosen arbitrarily close to $\varepsilon$ we obtain that $\Phi^{S}\left(\ell_{1}^{(2)}, Z_{\rho}^{(n)}, \varepsilon\right) \geqslant C(\varepsilon, \rho, n, \tilde{\theta})$ with $\tilde{\theta} \in[-1 / 2,1 / 2]$. Consequently, we have that $\Psi\left(\ell_{1}^{(2)}, Z_{\rho}^{(n)}\right) \geqslant \sqrt{\frac{2 \rho}{1-\rho+(2+\tilde{\theta}) / n}}$. When $n \rightarrow \infty$, we obtain the desired estimation $\Psi(\rho) \geqslant \sqrt{\frac{2 \rho}{1-\rho}}$.

4. Modified Bishop-Phelps-Bollobás moduli for operators. The following modification of the Bishop-Phelps-Bollobás theorem can be easily deduced from Proposition 2 just by substituting $\eta=\varepsilon, k=\sqrt{\varepsilon}$.

Theorem 8 (Modified Bishop-Phelps-Bollobás theorem). Let $X$ be a Banach space. Suppose $x \in B_{X}$ and $x^{*} \in B_{X^{*}}$ satisfy $x^{*}(x) \geqslant 1-\varepsilon(\varepsilon \in(0,2))$. Then there exists $\left(y, y^{*}\right) \in S_{X} \times X^{*}$ with $\left\|y^{*}\right\|=y^{*}(y)$ such that $\max \left\{\|x-y\|,\left\|x^{*}-y^{*}\right\|\right\} \leqslant \sqrt{\varepsilon}$.

The improvement in this estimate comparing to the original version appears because we do not demand $\left\|y^{*}\right\|=1$. It was shown in [11] that this theorem is sharp for a number of twodimensional spaces, which makes a big difference with the original Bishop-Phelps-Bollobás theorem, where the only (up to isometry) two-dimensional space, for which the theorem is sharp, is $\ell_{1}^{(2)}$. Bearing in mind this theorem it is natural to introduce the following quantities.

Definition 5. The modified Phelps-Bollobás modulus of a pair $(X, Y)$ is the function, which is determined by the following formula:

$\widetilde{\Phi}(X, Y, \varepsilon)=\inf \left\{\delta>0: \forall T \in B_{L(X, Y)}\right.$, if $x \in B_{X}$ and $\|T(x)\|>1-\varepsilon$, then there exist $y \in S_{X}$ and $F \in L(X, Y)$ satisfying $\|F(y)\|=\|F\|,\|x-y\|<\delta$ and $\left.\|T-F\|<\delta\right\}$.

The modified spherical Bishop-Phelps-Bollobás modulus of a pair $(X, Y)$ is the function, which is determined by the following formula:

$$
\begin{gathered}
\widetilde{\Phi^{S}}(X, Y, \varepsilon)=\inf \left\{\delta>0: \forall T \in S_{L(X, Y)}, \text { if } x \in S_{X} \text { and }\|T(x)\|>1-\varepsilon,\right. \text { then there exist } \\
\left.y \in S_{X} \text { and } F \in L(X, Y) \text { satisfying }\|T(y)\|=\|T\|,\|x-y\|<\delta \text { and }\|T-F\|<\delta\right\} .
\end{gathered}
$$

By analogy with Theorem 1 we prove the next result.

Theorem 9. Let $X$ and $Y$ be Banach spaces such that $Y$ has the property $\beta$ with parameter $\rho$. Then the pair $(X, Y)$ has the Bishop-Phelps-Bollobás property for operators and for any $\varepsilon \in(0,1)$

$$
\widetilde{\Phi^{S}}(X, Y, \varepsilon) \leqslant \widetilde{\Phi}(X, Y, \varepsilon) \leqslant \min \left\{\sqrt{\varepsilon} \sqrt{\frac{1+\rho}{1-\rho}}, 1\right\} .
$$

The proof is similar to that of Theorem 1 but it has some modifications and we give it here for the sake of clearness.

Proof. Consider $T \in B_{L(X, Y)}$ and $x \in B_{X}$ such that $\|T(x)\|>1-\varepsilon$ with $\varepsilon \in\left(0, \frac{1-\rho}{1+\rho}\right)$. Since $Y$ has the property $\beta$, there is $\alpha_{0} \in \Lambda$ such that $\left|y_{\alpha_{0}}^{*}(T(x))\right|>1-\varepsilon$. So, if we denote $x^{*}=T^{*} y_{\alpha_{0}}^{*}$, we have $x \in B_{X}, x^{*} \in B_{X^{*}}$ with $x^{*}(x)>1-\varepsilon$. We can apply formula (2) from Lemma 1 , for any $k \in(0,1)$. For every $\tilde{k} \in[\varepsilon, 1)$ let us take

$$
k=\frac{\tilde{k}\left(\left\|x^{*}\right\|-(1-\varepsilon)\right)}{\varepsilon\left\|x^{*}\right\|} .
$$


The inequality $\left\|x^{*}\right\| \geqslant x^{*}(x)>1-\varepsilon$ implies that $k>0$. On the other hand, $k=\tilde{k}\left(\frac{1}{\varepsilon}-\frac{(1-\varepsilon)}{\varepsilon\left\|x^{*}\right\|}\right) \leqslant$ $\tilde{k}\left(\frac{1}{\varepsilon}-\frac{(1-\varepsilon)}{\varepsilon}\right)=\tilde{k}<1$, so for this $k$ we can find $\zeta^{*} \in X^{*}$ and $z \in S_{X}$ such that there exist $z^{*} \in X^{*}$ and $z \in S_{X}$ such that $\left|z^{*}(z)\right|=\left\|z^{*}\right\|$ and

$$
\|x-z\|<\frac{1-\frac{1-\varepsilon}{\left\|x^{*}\right\|}}{k} \text { and }\left\|z^{*}-x^{*}\right\|<k\left\|x^{*}\right\| .
$$

For a real number $\eta$ satisfying $\eta>\frac{\rho\left(k\left\|x^{*}\right\|+\left\|x^{*}\right\| \cdot \mid 1-\left\|z^{*}\right\|\right)}{\left\|z^{*}\right\|(1-\rho)}$ we define the operator $S \in L(X, Y)$ by the formula

$$
S(t)=\left\|z^{*}\right\| T(t)+\left[(1+\eta) z^{*}(t)-\left\|z^{*}\right\| T^{*}\left(y_{\alpha_{0}}^{*}\right)(t)\right] y_{\alpha_{0}} .
$$

Let us estimate the norm of $S$. Recall that we denote $x^{*}=T^{*} y_{\alpha_{0}}^{*}$. Thus for all $y^{*} \in Y^{*}$,

$$
S^{*}\left(y^{*}\right)=\left\|z^{*}\right\| T^{*}\left(y^{*}\right)+\left[(1+\eta) z^{*}-\left\|z^{*}\right\| x^{*}\right] y^{*}\left(y_{\alpha_{0}}\right) .
$$

Since the set $\left\{y_{\alpha}^{*}: \alpha \in \Lambda\right\}$ is norming for $Y$ it follows that $\|S\|=\sup _{\alpha}\left\|S^{*} y_{\alpha}^{*}\right\|$.

$$
\|S\| \geqslant\left\|S^{*}\left(y_{\alpha_{0}}^{*}\right)\right\|=(1+\eta)\left\|z^{*}\right\| .
$$

On the other hand for $\alpha \neq \alpha_{0}$ we obtain

$$
\left\|S^{*}\left(y_{\alpha}^{*}\right)\right\| \leqslant\left\|z^{*}\right\|+\rho\left[\left\|z^{*}-x^{*}\right\|+\left\|x^{*}\right\| \cdot\left|1-\left\|z^{*}\right\|\right|+\eta\left\|z^{*}\right\|\right] \leqslant(1+\eta)\left\|z^{*}\right\| .
$$

Therefore, $\|S\|=\left\|S^{*}\left(y_{\alpha_{0}}^{*}\right)\right\|=\left\|z^{*}\right\|=\left|y_{\alpha_{0}}^{*}(S(z))\right| \leqslant\|S(z)\| \leqslant\|S\|$. So, we have $\|S\|=$ $\|S(z)\|=(1+\eta)\left\|z^{*}\right\|$.

Let us estimate $\|S-T\|$.

$$
\|S-T\|=\sup _{\alpha}\left\|S^{*} y_{\alpha}^{*}-T^{*} y_{\alpha}^{*}\right\|
$$

Notice also that $\left|1-\left\|z^{*}\right\|\right| \leqslant\left\|x^{*}-z^{*}\right\|+1-\left\|x^{*}\right\|<k\left\|x^{*}\right\|+1-\left\|x^{*}\right\|$. For $\alpha=\alpha_{0}$ we get

$$
\begin{gathered}
\left\|S^{*} y_{\alpha_{0}}^{*}-T^{*} y_{\alpha_{0}}^{*}\right\|=\left\|(1+\eta) z^{*}-x^{*}\right\| \leqslant\left\|z^{*}-x^{*}\right\|+\eta\left\|z^{*}\right\|< \\
<\frac{k\left\|x^{*}\right\|\left(1+\rho\left\|x^{*}\right\|\right)+\rho\left\|x^{*}\right\|\left(1-\left\|x^{*}\right\|\right)}{1-\rho} .
\end{gathered}
$$

Then $\|x-z\|<\frac{\varepsilon}{\hat{k}}$ and

$$
\left\|S^{*} y_{\alpha_{0}}^{*}-T^{*} y_{\alpha_{0}}^{*}\right\|<\frac{\tilde{k} \frac{\left\|x^{*}\right\|-(1-\varepsilon)}{\varepsilon}\left(1+\rho\left\|x^{*}\right\|\right)+\rho\left\|x^{*}\right\|\left(1-\left\|x^{*}\right\|\right)}{1-\rho} \leqslant \frac{\tilde{k}(1+\rho)}{1-\rho} .
$$

The latter inequality holds, because if we consider the function

$$
f(t)=\frac{\tilde{k} \frac{t-(1-\varepsilon)}{\varepsilon}(1+\rho t)+\rho t(1-t)}{1-\rho}
$$

with $t \in(1-\varepsilon, 1)$, then $f^{\prime} \geqslant 0$, so $\max f=f(1)=\frac{\tilde{k}(1+\rho)}{1-\rho}$. For $\alpha \neq \alpha_{0}$ we obtain

$$
\begin{gathered}
\left\|S^{*} y_{\alpha}^{*}-T^{*} y_{\alpha}^{*}\right\| \leqslant\left|1-\left\|z^{*}\right\|\right|+\rho\left(\left\|z^{*}-x^{*}\right\|+\left\|x^{*}\right\| \cdot\left|1-\left\|z^{*}\right\|\right|+\eta\left\|z^{*}\right\|\right)< \\
<k\left\|x^{*}\right\|+1-\left\|x^{*}\right\|+\frac{\rho}{1-\rho}\left[k\left\|x^{*}\right\|-\rho k\left\|x^{*}\right\|+\left\|x^{*}\right\| \cdot\left|1-\left\|z^{*}\right\|\right|-\right. \\
\left.-\rho\left\|x^{*}\right\| \cdot\left|1-\left\|z^{*}\right\|\right|+\rho k\left\|x^{*}\right\|+\rho\left\|x^{*}\right\| \cdot\left|1-\left\|z^{*}\right\|\right|\right] \leqslant \\
\leqslant k\left\|x^{*}\right\|+1-\left\|x^{*}\right\|+\frac{\rho}{1-\rho}\left[k\left\|x^{*}\right\|+\left\|x^{*}\right\| \cdot\left(k\left\|x^{*}\right\|+1-\left\|x^{*}\right\|\right)\right] .
\end{gathered}
$$


Substituting the value of $k$ we get

$$
\begin{aligned}
\left\|S^{*} y_{\alpha}^{*}-T^{*} y_{\alpha}^{*}\right\| & <\frac{1-\rho\left(1-\left\|x^{*}\right\|\right)}{1-\rho}\left[\tilde{k} \frac{\left\|x^{*}\right\|-(1-\varepsilon)}{\varepsilon}+1-\left\|x^{*}\right\|\right]+ \\
& +\frac{\rho \tilde{k}}{1-\rho} \frac{\left\|x^{*}\right\|-(1-\varepsilon)}{\varepsilon} \leqslant \frac{\tilde{k}(1+\rho)}{1-\rho} .
\end{aligned}
$$

To get the latter inequality we again use the fact that the function

$$
f_{1}(t)=\frac{1-\rho(1-t)}{1-\rho}\left[\tilde{k} \frac{t-(1-\varepsilon)}{\varepsilon}+1-t\right]+\frac{\rho \tilde{k}}{1-\rho} \frac{t-(1-\varepsilon)}{\varepsilon}
$$

is increasing if $\tilde{k} \geqslant \varepsilon$, so $\max f_{1}=f_{1}(1)=\frac{\tilde{k}(1+\rho)}{1-\rho}$. So, $\|T-S\| \leqslant \frac{\tilde{k}(1+\rho)}{1-\rho}$.

Let us substitute $\tilde{k}=\sqrt{\frac{\varepsilon(1-\rho)}{1+\rho}}$ (here we need $\varepsilon<\frac{1+\rho}{1-\rho}$ which holds for any $\varepsilon \in(0,1)$ and also $\varepsilon<\frac{1-\rho}{1+\rho}$ ). Then we obtain

$$
\max \{\|z-x\|,\|T-S\|\} \leqslant \sqrt{\frac{\varepsilon(1+\rho)}{1-\rho}}
$$

Finally, if $\varepsilon \geqslant \frac{1-\rho}{1+\rho}$, we can always approximate $(x, T)$ by the same point and zero operator, so $\max \{\|z-x\|,\|T-S\|\} \leqslant 1$.

The above theorem implies that if $\beta(Y)=0$, then $\widetilde{\Phi^{S}}(X, Y, \varepsilon) \leqslant \widetilde{\Phi}(X, Y, \varepsilon) \leqslant \sqrt{\varepsilon}$. We are going to demonstrate that this estimation is sharp for $X=\ell_{1}^{(2)}, Y=\mathbb{R}$.

Theorem 10. $\widetilde{\Phi^{S}}\left(\ell_{1}^{(2)}, \mathbb{R}, \varepsilon\right)=\widetilde{\Phi}\left(\ell_{1}^{(2)}, \mathbb{R}, \varepsilon\right)=\sqrt{\varepsilon}, \varepsilon \in(0,1)$.

Proof. We must show that for every $0<\varepsilon<1$ and for every $\delta>0$ there is a pair $\left(x, x^{*}\right)$ from $\Pi_{\varepsilon}^{S}\left(\ell_{1}^{(2)}, \mathbb{R}\right)$ such that for every pair $\left(y, y^{*}\right) \in S_{\ell_{1}^{(2)}} \times \ell_{\infty}^{(2)}$ with $\left|y^{*}(y)\right|=\left\|y^{*}\right\|$

$$
\max \left\{\|x-y\|,\left\|x^{*}-y^{*}\right\|\right\} \geqslant \sqrt{\varepsilon}-\delta \text {. }
$$

Fix $\varepsilon_{0}<\varepsilon$ such that $\sqrt{\varepsilon_{0}}>\sqrt{\varepsilon}-\delta$. Take the point $x:=\left(1-\frac{\sqrt{\varepsilon_{0}}}{2}\right) e_{1}+\left(\frac{\sqrt{\varepsilon_{0}}}{2}\right) e_{2}$, and the functional $x^{*}(z):=z_{1}+\left(1-2 \sqrt{\varepsilon_{0}}\right) z_{2}$. Notice that $x^{*}(x)=1-\varepsilon_{0}>1-\varepsilon$.

Consider the set $U$ of those $y \in S_{X}$ that $\|x-y\|<\sqrt{\varepsilon_{0}}$. $U$ is the intersection of $S_{X}$ with the open ball of radius $\sqrt{\varepsilon_{0}}$ centered in $x$. As $\left\|x-e_{1}\right\|=\sqrt{\varepsilon_{0}}$, and $\left\|x-e_{2}\right\|=2-\sqrt{\varepsilon_{0}} \geqslant \sqrt{\varepsilon_{0}}$, so, $U \subset] e_{1}, e_{2}\left[\right.$, and for every $y=a e_{1}+b e_{2} \in U a>0$ and $b>0$.

Assume that $\left|y^{*}(y)\right|=\left\|y^{*}\right\|$ for some $y \in U$ and $\left\|x^{*}-y^{*}\right\| \leqslant \sqrt{\varepsilon_{0}}$. Then we are forced to have $y^{*}=\left(y^{*}\left(e_{1}\right), y^{*}\left(e_{2}\right)\right)$, where $\left|y^{*}\left(e_{1}\right)\right|=\left|y^{*}\left(e_{2}\right)\right|$ and $y^{*}\left(e_{1}\right) \cdot y^{*}\left(e_{2}\right) \geqslant 0$. Notice that

$$
\begin{gathered}
\left|x^{*}\left(e_{1}\right)-y^{*}\left(e_{1}\right)\right|=\left|1-y^{*}\left(e_{1}\right)\right| \leqslant\left\|x^{*}-y^{*}\right\| \leqslant \sqrt{\varepsilon} \Rightarrow y^{*}\left(e_{1}\right) \geqslant 1-\sqrt{\varepsilon_{0}}, \\
\left|x^{*}\left(e_{2}\right)-y^{*}\left(e_{2}\right)\right|=\left|1-2 \sqrt{\varepsilon_{0}}-y^{*}\left(e_{2}\right)\right| \leqslant\left\|x^{*}-y^{*}\right\| \leqslant \sqrt{\varepsilon_{0}} \Rightarrow y^{*}\left(e_{2}\right) \leqslant 1-\sqrt{\varepsilon_{0}} .
\end{gathered}
$$

Then $y^{*}=\left(1-\sqrt{\varepsilon_{0}}, 1-\sqrt{\varepsilon_{0}}\right)$, so $\left\|x^{*}-y^{*}\right\|=\sqrt{\varepsilon_{0}}>\sqrt{\varepsilon}-\delta$. It follows that inequality (16) holds, as desired.

Also with the same space $Y=Y_{\rho}$ equipped with the norm (10) we have an estimation from below which almost coincides with estimation (15) from above for values of $\rho$ close to 1 . 
Theorem 11. Let $\rho \in[1 / 2,1), 0<\varepsilon<1$. Then, for the space $Y=Y_{\rho}$ one has

$$
\widetilde{\Phi^{S}}\left(\ell_{1}^{(2)}, Y, \varepsilon\right) \geqslant \min \left\{\sqrt{\varepsilon} \sqrt{\frac{2 \rho}{1-\rho}}, 1\right\}
$$

\section{An open problem.}

Problem 1. Is it true, that $\Phi^{S}(X, \mathbb{R}, \varepsilon) \leqslant \min \{\sqrt{2 \varepsilon}, 1\}$ for all real Banach spaces $X$ ?

In order to explain what do we mean, recall that for the original Bishop-Phelps-Bollobás modulus the estimation

$$
\Phi_{X}^{S}(\varepsilon) \leqslant \sqrt{2 \varepsilon}
$$

holds true for all $X$. In other words, for every $\left(x, x^{*}\right) \in S_{X} \times S_{X^{*}}$ with $x^{*}(x)>1-\varepsilon$, there is $\left(y, y^{*}\right) \in S_{X} \times S_{X^{*}}$ with $y^{*}(y)=1$ such that $\max \left\{\|x-y\|<\sqrt{2 \varepsilon},\left\|x^{*}-y^{*}\right\|\right\}<\sqrt{2 \varepsilon}$.

When we take $Y=\mathbb{R}$ in the definition of $\Phi^{S}(X, Y, \varepsilon)$, the only difference with $\Phi_{X}^{S}(\varepsilon)$ is that by attaining norm we mean $\left|y^{*}(y)\right|=1$, instead of $y^{*}(y)=1$. So, in the case of $\Phi^{S}(X, \mathbb{R}, \varepsilon)$ we have more possibilities to approximate $\left(x, x^{*}\right) \in S_{X} \times S_{X^{*}}$ with $x^{*}(x)>1-\varepsilon$ :

$$
\left(y, y^{*}\right) \in S_{X} \times S_{X^{*}} \operatorname{with} y^{*}(y)=1 \text { or } y^{*}(y)=-1 .
$$

Estimation (17) is sharp for the two-dimensional real $\ell_{1}$ space: $\Phi_{\ell_{1}^{(2)}}^{S}(\varepsilon)=\sqrt{2 \varepsilon}$, but, as we have shown in Theorem $4 \Phi^{S}\left(\ell_{1}^{(2)}, \mathbb{R}, \varepsilon\right)=\min \{\sqrt{2 \varepsilon}, 1\}$.

Estimations $\Phi_{\ell_{1}^{(2)}}^{S}(\varepsilon)=\sqrt{2 \varepsilon}$, and $\Phi^{S}\left(\ell_{1}^{(2)}, \mathbb{R}, \varepsilon\right)=\min \{\sqrt{2 \varepsilon}, 1\}$. coincide for $\varepsilon \in(0,1 / 2)$, but for bigger values of $\varepsilon$ there is a significant difference. We do not know whether the inequality $\Phi^{S}(X, \mathbb{R}, \varepsilon) \leqslant \min \{\sqrt{2 \varepsilon}, 1\}$ holds true for all $X$.

Moreover, in all examples that we considered we always were able to estimate $\Phi^{S}(X, Y, \varepsilon)$ from above by 1 . So, we don't know whether the statement of Theorem 1 can be improved to

$$
\Phi^{S}(X, Y, \varepsilon) \leqslant \min \left\{\sqrt{2 \varepsilon} \sqrt{\frac{1+\rho}{1-\rho}}, 1\right\} .
$$

Acknowledgment. The research of the first author is done in frames of Ukranian Ministry of Science and Education Research Program 0115U000481. The research of second named author has been partially performed during her stay in University of Murcia in frames of Erasmus + program. The authors are thankful to the anonymous referee for his (her) helpful suggestions.

\section{REFERENCES}

1. Acosta M.D., Aron, R.M., García D., Maestre M. The Bishop-Phelps-Bollobás Theorem for operators, J. Funct. Anal., 254 (2008), 2780-2799.

2. Acosta M.D., Guerrero B., García J., Kim S.K., Maestre, M. Bishop-Phelps-Bollobás property for certain spaces of operators, J. Math. Anal. Appl., 414 (2014), №2, 532-545. 
3. Bishop, E., Phelps, R.R. A proof that every Banach space is subreflexive, Bull. Amer. Math. Soc., 67 (1961), 97-98.

4. Bollobás, B. An extension to the theorem of Bishop and Phelps, Bull. London Math. Soc., 2 (1970), 181-182.

5. Chica M., Kadets V., Martín M., Moreno-Pulido S., Rambla-Barreno F. Bishop-Phelps-Bollobás moduli of a Banach space, J. Math. Anal. Appl., 412 (2014), №2, 697-719.

6. Chica M., Kadets V., Martín M., Merí J., Soloviova M. Two refinements of the Bishop-Phelps-Bollobás modulus, Banach J. Math. Anal., 9 (2015), №4, 296-315.

7. Chica M., Kadets V., Martín M., Merí J. Further properties of the Bishop-Phelps-Bollobás moduli, Mediterranean Journal of Mathematics, 13(5) (2016), 3173-3183.

8. Diestel J., Geometry of Banach spaces, Lecture notes in Math., V.485, Springer-Verlag, Berlin, 1975.

9. James R.C. Uniformly non-square Banach spaces, Ann. Math., 80(2) (1964), 542-550.

10. Partington J., Norm attaining operators, Israel J. Math., 43 (1982), 273-276.

11. Kadets V., Soloviova M., A modified Bishop-Phelps-Bollobás theorem and its sharpness, Mat. Stud., 44 (2015), 84-88.

12. Lindenstrauss, J., On operators which attain their norm, Israel J. Math., 1 (1963), 139-148.

13. Phelps R.R., Support cones in Banach spaces and their applications, Adv. Math., 13 (1974), 1-19.

School of Mathematics and Informatics

Kharkiv V.N. Karazin National University

vova1kadets@yahoo.com

mariiasoloviova93@gmail.com

Received 5.01.2017 